\title{
The Influence of Adverse Childhood Experiences (ACEs), Including the COVID-19 Pandemic, and Toxic Stress on Development and Health Outcomes of Latinx Children in the USA: a Review of the Literature
}

\author{
Natalie Claypool ${ }^{1}$. Arelis Moore de Peralta ${ }^{2}$
}

Accepted: 27 June 2021 / Published online: 11 July 2021

(c) The Author(s), under exclusive licence to Springer Nature Switzerland AG 2021

\begin{abstract}
The purpose of this review is to synthesize existing literature to analyze the influence of the Adverse Childhood Experiences (ACEs), including the COVID-19 pandemic, and toxic stress on child development and lifelong health outcomes of Latinx children in the USA, utilizing the ACE framework. Without adequate protective factors, children's early experiences with adversity and toxic stress have implications for their physiological, psychological, and social health. The COVID19 pandemic has shown to exacerbate childhood adversity and toxic stress and has disproportionately harmed Latinx communities. In applying the ACE framework to US-Latinx populations, relevant findings concerning a potential failure of ACEs to accurately capture Latinx experiences of adversity were highlighted, as well as the need to classify the COVID-19 pandemic as an ACE. Research suggest that firstgeneration Latinx immigrants report lower-than-average rates of ACEs despite the various disparities ethnic minorities face in the USA. A discussion on whether this health paradox arises because of the failure of ACEs to properly identify adverse experiences unique to immigrants or if it is related with immigrant families' protective cultural factors. The compounding experiences of discrimination, immigration anxieties, and now also pandemic-related hardship that have the potential to harm Latinx children's cognitive, emotional, and physical development were highlighted. Evidence-based interventions that were discussed in this report include promotion of resiliency through healthy adult relationships, policies that screen for ACEs early on in a child's life, trauma-informed care and innovative treatment programs, and strengthening existing protective services through financial and political support.
\end{abstract}

Keywords Adverse Childhood Experiences · Toxic stress · COVID-19 - Latinx communities $\cdot$ Child development $\cdot$ Health outcomes

Arelis Moore de Peralta ared@clemson.edu

Extended author information available on the last page of the article 


\section{Introduction}

Childhood experiences, both positive and negative, shape the physical, mental, and social health of individuals throughout their entire lives. Early childhood adversity and its residual effects of toxic stress are shown to impact both early development and long-term health outcomes well into adulthood (Cittern et al., 2018; Espeleta et al., 2018; Flores \& Salazar, 2017; Grey et al., 2019; McKelvey et al., 2019; Mersky et al., 2013; National Scientific Council on the Developing Child, 2020). The purpose of this literature review is to synthesize existing literature to analyze the influences of Adverse Childhood Experiences (ACEs), including the COVID-19 pandemic context as an ACE, and toxic stress on child development and lifelong health outcomes of Latinx children in the USA and its adverse impacts on Latinx communities.

Negative events of adversity and trauma experienced in early childhood can be identified, counted, and examined through the framework of ACEs. In this study, ACEs are defined as "traumatic events of childhood that include physical, sexual, and emotional abuse, as well as the experience of household dysfunction, such as divorce/separation, domestic violence, or living with an adult experiencing mental illness, substance use/misuse, or incarceration," with childhood being defined as taking place in the first 18 years of life (Crouch et al., 2019, pp.209). ACEs fall under the three categories of abuse, household challenges, and neglect (Felitti et al., 1998). Specific ACEs include the following ten experiences: physical abuse, emotional abuse, sexual abuse, physical neglect, emotional neglect, caregiver(s) with mental illness, maternal violence, divorce or separation of parents, household substance abuse, and incarcerated caregiver(s) (Felitti et al., 1998). These experiences can be quantified into the following three scores of no ACEs (zero of these experiences in the first 18 years of life), low ACEs (one of these experiences in the first 18 years of life), and high ACEs (two or more of these experiences in the first 18 years of life) (Crouch et al., 2019). These categories no, low, and high ACEs are used to quantify the variety of adversity an individual experienced in childhood.

In applying the ACE framework to Latinx populations in the USA, some relevant findings concerning childhood adversity expand beyond the parameters of ACEs (Felitti et al., 1998). The specific ten experiences defined by ACEs may not be entirely inclusive of all adverse events and conditions impactful to child development (Ellis \& Dietz, 2017). There is existing literature that explores limitation in the ACE framework specifically in its application to Latinx populations (Flores \& Salazar, 2017). An additional parameter to measure childhood adversity is applied through the consideration of toxic stress. Toxic stress is defined as prolonged activation of the sympathetic nervous system without proper protective factors to provide relief, and it is applied thus forth as a potential consequence of ACEs (National Scientific Council on the Developing Child, 2005/2014). Experiencing adversity without protective factors can influence physiological, psychological, and social well-being through this excessive, unrelieved stress (National Scientific Council on the Developing Child, 2005/2014). As important findings of 
the effects of childhood adversity may extend beyond the specific ten experiences defined by ACEs, relevant research and findings mentioning toxic stress, but not explicitly ACEs, are applied in various sections of this study to be more inclusive of the Latinx experience in the USA. The COVID-19 context and realities can represent an ACE in that it has amplified toxic stress and risk of experiencing the 10 ACEs due to pandemic-related stressors (Sanders, 2020).

Variations have been found in types of adverse experiences reported across sociodemographic categories (Felitti et al., 1998). For example, children who were older, non-Hispanic African Americans, having special healthcare needs and living in poverty or rural areas, were more likely to be exposed to parental divorce/separation than the average population (Crouch et al, 2019). These study findings reflected that there is significant diversity in types of adversity experienced in childhood, but nonetheless, adversity is a widespread experience. While there is existing literature that explores racial and ethnic disparities in ACEs and how these disparities impact health outcomes (Maguire-Jack et al., 2020), data comparisons within Latinx communities, particularly between immigrant and nonimmigrant children, are lacking. The term Latinx is used to refer to those of Latin American origin or descent by using a gender-neutral alternative to Latino or Latina (Noe-Bustamante et al., 2020). The population of Latinx individuals born in the USA is expected to grow by $122 \%$ by 2060 , and the population of Latinx immigrant is expected to grow by $38.6 \%$ by 2060 (Vespa et al., 2020). The total percentage of Latinx children is expected to make up 31.9\% of the total population of US children by 2060 (Vespa et al., 2020). The lack of emphasis on the experiences of and challenges faced by diverse populations leads to inaccuracies in key data concerning childhood adversity among a significant proportion of the USA and stalemates regarding effective interventions to prevent and negate the harm of ACEs for all. This review seeks to bridge this gap by emphasizing the differences in exposure rates and outcomes within various subsets of Latinx communities.

This study includes a portrayal of the COVID-19 pandemic as an additional ACE by identifying in the literature the mechanisms through which the pandemic has impacted the health and well-being of Latinx populations in the USA, particularly by exacerbating preexisting health and other related inequities in this population (Parolin, 2021; Raviv et al., 2021). Latinx communities have faced disproportionate impacts in regarding infection rates, access to care, economic hardship, unemployment, and deaths (Branson-Potts et al., 2020; Capps \& Fix, 2020; Cooper \& Hanlon, 2020; Gelatt et al., 2020; Passel \& D’Vera, 2011). These impacts are clearly demonstrated through quantitative statistics such as the greater proportion of COVID-19 cases among Latinx individuals in comparison the Latinx proportion of the total US population, immigrant women's disproportionate rates of unemployment, and the overwhelming percentage of essential workers being Latinx (Branson-Potts et al., 2020; Cooper \& Hanlon, 2020; Gelatt et al., 2020). Further, undocumented Latinx immigrants in particular experience healthcare struggles because of barriers to government aid, lack of health insurance, and threats posed by immigration services (Passel \& D'Vera, 2011).

The first inquiry guiding this narrative review of relevant literature was what are the unique ACEs (including the COVID-19 pandemic), and toxic stress, faced 
by Latinx communities in the USA that influence child development and lifelong health outcomes? The second inquiry was, how do ACEs (including the COVID19 pandemic), and toxic stress, impact child development and lifelong health outcomes? The first outcome variable of interest is child development, which refers to the course of physical, cognitive, emotional, and language growth that occurs in the first 18 years of life (Center on the Developing Child, 2007). The second outcome variable of interest is health outcomes, referring to one's mental, physical, and social well-being (Center on the Developing Child, 2007). Thus, literature concerning the impact of ACEs upon child development and health outcomes in Latinx communities was considered relevant to the questions guiding this paper. The contents of this literature review were organized as follows: ACE specific to Latinx communities in the USA, including toxic stress, and a consideration of the special context imposed by the COVID-19 pandemic as an additional ACE, Latinx ACEs as a health paradox, Latinx families' protective factors, and consequences of ACEs in child development and lifelong health. Finally, selected evidence-based interventions from the literature are explored in the results/discussion section.

\section{Methods}

The methodology of this narrative literature review has been displayed within the Prisma Flow Diagram in Fig. 1. The process included an identification of the research objective, formulation of two research questions, development and execution of a search strategy, selection and critical appraisal of studies, abstraction and synthesis of data, and assessment of the studies' quality and biases.

This narrative literature review analyzes scientific articles from peer-reviewed psychology and health journals, news reports for current event analyses, and technical publications from government and international cooperation agencies. Articles were found using EBSCOHost, PsycInfo, and PubMed, and additional sources were utilized from the reference lists of revised articles as well as the authors' personal and academic collections. Using search engines, a variety of conjugated search terms were used to find relevant articles, such as "adverse childhood experiences," "toxic stress," "development," "health outcomes," "Latinx," "Hispanics," "United States," "COVID-19," "SARS-CoV-2," and "coronavirus," as well as a variety of additional MeSH terms for unmentioned variations of the aforementioned terms. The MeSH terms used are included within Fig. 2, as well as the number of articles found within each conjugation. Other relevant articles concerning current event analyses were collected from news reports and technical publications from government and international cooperation agencies. All additional publications were identified from the articles' reference lists and the authors personal files.

Literature relevant to the impact of ACEs upon child development and health outcomes in Latinx communities was considered relevant to the questions guiding this paper. The inclusion criteria for the works cited were the most recent and relevant information on ACEs and Latinx communities in the USA. The accepted period for publications was from 2000 to 2020, with an exception being the seminal ACE study executed in 1998 (Felitti et al., 1998). Another inclusion criterium 


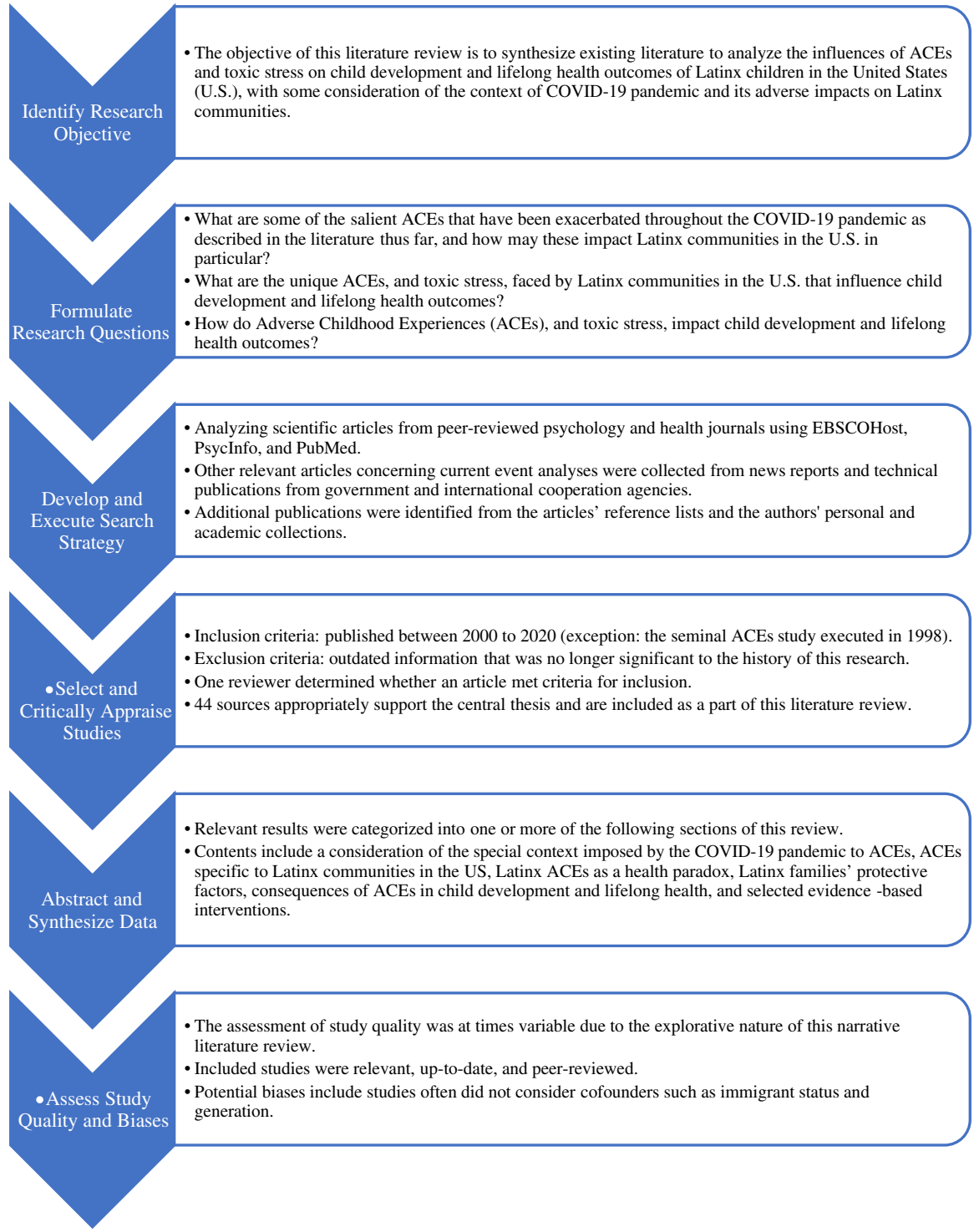

Fig. 1 Flowchart summarizing the process followed for the narrative literature review

was that all articles were written in English. The exclusion criteria include any outdated information that was no longer significant to the history of this research. One reviewer determined whether an article met criteria for inclusion. One hundred seventy-two sources were reviewed, and 25 of those were determined to appropriately address the central inquiries and thus included as a part of this literature review. Seventeen other sources were included from reference lists and the authors' personal files. In total, 42 sources were determined to appropriately 


\section{5 results}

- "child development OR child wellbeing OR child well-being OR child adjustment" AND "health outcomes OR health impacts OR health effects OR effects on health OR health consequences" AND "Latinx OR Latino OR Latina OR Hispanic" AND "United States OR USA OR U.S. OR America"

\section{2 results}

- "adverse childhood experiences OR ACEs OR toxic stress OR childhood trauma" AND "health outcomes OR health impacts OR health effects OR effects on health OR health consequences" AND "Latinx OR Latino OR Latina OR Hispanic" AND "United States OR USA OR U.S. OR America"

\section{7 results}

- "adverse childhood experiences OR ACEs OR toxic stress OR childhood trauma" AND "child development OR child wellbeing OR child well-being OR child adjustment" AND "Latinx OR Latino OR Latina OR Hispanic" AND "United States OR USA OR U.S. OR America"

\section{3 results}

• "COVID -19 OR coronavirus OR 2019-NCOV OR SARS-COV-2 OR COV-19" AND "child development OR child wellbeing OR child well-being OR child adjustment" AND "Latinx OR Latino OR Latina OR Hispanic"

\section{Total results: 177}

Fig. 2 Database results of the search strategy

support the central thesis and included as a part of this literature review. All included sources can be found in the references section.

After the articles were collected, relevant results were categorized into one or more of the following sections of this review. The contents of this literature review were organized as follows: a conceptualization of ACEs and toxic stress, a consideration of the special context imposed by the COVID-19 pandemic as an additional ACE, ACEs specific to Latinx communities in the USA, Latinx ACEs as a health paradox, Latinx families' protective factors, and consequences of ACEs in child development and lifelong health. Finally, selected evidence-based interventions from the literature are presented.

The assessment of study quality was at times variable due to the explorative nature of this narrative literature review. Included studies were ensured to be of high quality by only including relevant, up-to-date, and peer-reviewed (in the case of empirical data) articles. Biases that led to the exclusion of studies include those with small sample sizes or those of which did not appropriately consider potential confounders. However, some included studies did not consider potential confounders such as immigrant or generation status, but given the limited number of studies published concerning these topics, some of these articles were included if the article presented other strengths.

After reviewing the 177 articles, 25 of these were considered suitable and included in the literature review. Seventeen additional sources were found from reference lists, and the authors' personal files for a total of 42 included sources. 


\section{Results/Discussion of the Literature Review}

\section{ACEs Specific to Latinx Communities in the USA}

Beyond the recent experiences facilitated by the pandemic, ACEs can be further analyzed in their pertinence to Latinx communities. Research shows that the prevalence and types of ACEs can vary among sociodemographic factors (Crouch et al, 2019). Regarding race and ethnicity, white children are found to have a lower total number of ACEs and less exposure to specific ACEs in comparison to Latinx children (Maguire-Jack et al., 2020). When looking at the intersection of ethnicity and class, the prevalence of ACEs in low-income Latinx children is similar to the prevalence for low-income children in other ethnic groups (Crouch et al., 2019). Conversely, the prevalence of ACEs is significantly higher in nonimmigrant Latinx children as compared to immigrant Latinx children (Loria \& Caughy, 2018). These findings from the Crouch et al. and Loria and Caughy studies suggest that differences in ACEs prevalence are most apparent when comparing children of higher-income families and of different immigration statuses.

Further differences arise when analyzing the prevalence of ACEs within the diverse Latinx population. The overall prevalence rates of ACEs within various subgroups of low-income Latinx children show significant variation, particularly regarding immigrant families. Immigrant families make up a significant proportion of children's at-home lives as $25.8 \%$ of children in the USA were from families with at least one immigrant parent in 2019 (Vespa et al., 2020). While not all immigrants within the USA are of Latinx ethnicity, $44.8 \%$ of the 44 million immigrants in the USA were Latinx in 2016, and it is estimated that most immigrants are from Latin America and the Caribbean (Vespa et al., 2020). Latinx children in immigrant families were significantly less likely to experience a high number of ACEs (16\%) than Latinx children in nonimmigrant families (30\%) (Caballero et al., 2017). Only 13\% of immigrant Latinx children were shown to experience two or more ACEs in comparison to $40 \%$ of nonimmigrant Latinx children (Loria \& Caughy, 2018). Immigrant and non-immigrant children showing diverse experiences of adversity suggests a wide variation of experiences within Latinx communities.

ACE prevalence variations between immigrant and nonimmigrant Latinx children is further explored in this study through stablishing differences by immigrant generational status. Among nonimmigrant Latinx children, the prevalence of exposure to adversity was highest among those who were third or higher-generation immigrants and lowest among those who were second-generation immigrant children (Loria \& Caughy, 2018). These differences in generation status apply to both the total and mean number of ACEs, and they persist even after stratifying participants by variations in their federal poverty level (FPL) and age (Loria \& Caughy, 2018). In the USA, socioeconomic status and ethnicity are strongly associated with health and lifespan (Van Hook et al., 2013) leading to an assumption that Latinx immigrants would have higher reports of adversity and poor health outcomes. However, this relationship seems opposite to the Latinx 
immigrant children's low reports of adversity observed in the USA. One interpretation of these differences is a health paradox in relation to ACEs among Latinx immigrants.

\section{The COVID-19 Pandemic as an ACE}

The COVID-19 pandemic has exacerbated ACEs, which is demonstrated through Table 1's depiction of the 10 ACEs and how they have changed in frequency, intensity, and manifestation throughout the duration of the pandemic (Table 1) (APA, 2020; Bryant et al., 2020; Chowdhury, 2020; Lawson et al., 2020; Panchal et al., 2020; Parolin, 2021; Raviv et al., 2021; Taub, 2020; Tener et al., 2020; Zaami et al., 2020).

The impact of the COVID-19 pandemic-imposed restrictions and challenges including social isolation, economic downturn, health anxieties, loss of family members, school closings, and more are themselves incidences of trauma in children's lives (Sanders, 2020). Evidence already shows that specific ACEs have increased in their frequency during the pandemic (Table 1). Rates of intimate partner violence have risen significantly, and children witnessing domestic abuse are one of the ten ACEs (Bryant et al., 2020). The additional ACEs of parental substance abuse and mental health issues have also heightened (Panchal et al., 2020). Pre-pandemic research had found that social isolation and low socioeconomic statuses are major risk factors for ACEs, and the pandemic has significantly worsened both factors (Bryant et al., 2020). Social isolation can lead to increased risk for depression and anxiety (Cooper \& Hanlon, 2020). These stressors have increased experiences of adversity and stress within families, and children pick up on their parents' anxieties concerning food, housing, and job insecurity (Sanders, 2020).

Past recessions of great economic insecurity give insights into what can be expected from these financial stressors including food, housing, and job insecurity. During the 2008 Great Recession, Google searches about parental abuse and on how to report these incidents skyrocketed, while reports to child protective services (CPS) declined (Bryant et al., 2020). Decreased reports to CPS are indicative of child abuse persisting, rather than declining, as children receive less intervention from teachers, physicians, and well-child visits that allow abuse to be screened for (Cooper \& Hanlon, 2020). Further, during the Great Recession, the rates of child deaths rose in the states most economically impacted by the recession (Bryant et al., 2020). Studies showed that over $78 \%$ of the abuse and neglect that children experience is perpetrated by their parents (Bryant et al., 2020), and children are now isolated at home without their usual support networks of teachers, peers, extended family, and coaches.

The COVID-19 pandemic has disproportionately impacted low-income and minority populations, both directly through higher exposure rates and increased susceptibility due to high rates of pre-existing chronic conditions and indirectly through less financial and social stability (Sanders, 2020). Significant differences in exposure to the virus have been observed across strata related to both ethnicity and household income (Raviv et al., 2021). Higher rates of exposure resulted from high levels of 


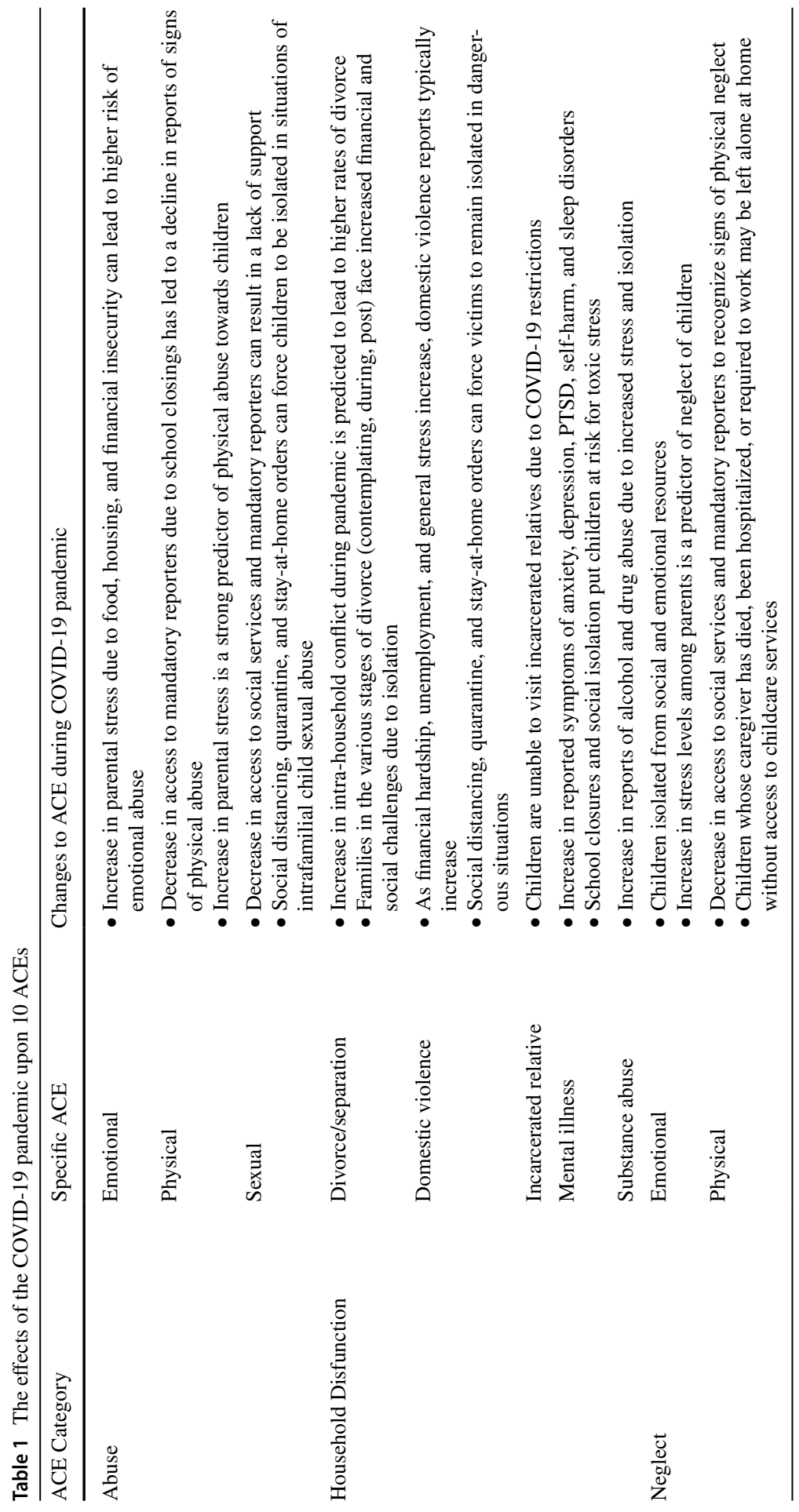


employment in essential services, a lack of paid sick, and residing in closer quarters (National Scientific Council of the Developing Child, 2020). These disparities are more deeply rooted in structural inequities like residential segregation, food insecurity, and poor environmental health as a result of decades of discriminatory policies and marginalization (National Scientific Council of the Developing Child, 2020). For example, $75 \%$ of black and Latinx children who experienced food insecurity throughout the pandemic had already felt its effects prior to the pandemic's onset (Parolin, 2021).

The interaction between childhood adversity and health outcomes is a key component of COVID-19 disparities' impacts. Jack P. Shonkoff and David R. Williams of the National Scientific Council on the Developing Child (2020, pp.1) posited that "the structural legacies of racism and other cross-generational traumas may be linked to levels of chronic stress that increase susceptibility to the kinds of health impairments that result in greater risk of harm from COVID-19." The council report stated that the origins of the diseases that make one more susceptible to COVID19 (COPD, hypertension, obesity, heart disease, diabetes, etc.) are associated with impairment to the developing immune system as a result of significant adversity experienced during the prenatal period until 3 years after birth. Studies show that individuals with a history of ACEs are more vulnerable to the stressors resulting from COVID-19 (Cooper \& Hanlon, 2020).

Latinx communities have shown to be disproportionately affected in the COVID-19 pandemic regarding infection rates, economic hardship, unemployment, and deaths (Capps \& Fix, 2020). In many states, the proportion of Latinx positive COVID-19 cases is much higher than their proportion of the total population (Branson-Potts et al., 2020). Immigrant women have faced the highest rates of unemployment, with employment rates falling from $53 \%$ to $46 \%$, throughout the pandemic due to difficulties in balancing work and at-home schooling of children (Gelatt et al., 2020). One-third of jobs lost in the USA were within the immigrant women-dominated industry of leisure and hospitality (Gelatt et al., 2020). Essential workers are overwhelmingly Latinx, leading to higher rates of anxieties related to potential exposure and the challenges of finding childcare (Cooper \& Hanlon, 2020). In California, Latinx workers consist of over half of employees in the food service, construction, and agriculture industries, and only $42 \%$ of Latinx workers reported that working from home was an option in comparison to $61 \%$ of white respondents (Branson-Potts et al., 2020). Further, undocumented Latinx immigrants are unable to seek government financial resources or proper health care if infected without health insurance (Passel and D'Vera, 2011). Even documented immigrants may experience fear of immigration services and avoid seeking these resources altogether (Passel and D'Vera, 2011).

Though empirical studies that detail how the COVID-19 pandemic has exacerbated ACEs specific to Latinx children are limited, some of Table 1 findings could be applied explicitly to this demographic. During the pandemic, Latinx youth have been disproportionately exposed to caregiver's illness, death, and worsening mental health, as well as more household dysfunction resulting from stay-at-home orders and social isolation (Raviv et al., 2021). Statistics have shown that Latinx individuals were more likely to die of COVID-19 early on in 
the pandemic, leaving Latinx children at higher risk for neglect (Parolin, 2021). Throughout the pandemic, Latinx children were more likely to experience poverty, food insufficiency, parental job loss, and school closures, thus experiencing higher risk for abuse, household dysfunction, and neglect (Parolin, 2021). Undocumented immigrants were unable to receive government relief stimulus checks throughout the pandemic, further worsening the economic disparity (Parolin, 2021). The compounding experiences of pandemic-related hardship, discrimination, and immigration anxieties have likely worsened the already concerning previous reports of the state of Latinx immigrant mental health (Capps \& Fix, 2020). COVID-19's impacts upon economic, social, emotional, and physical health has the ability to persist for years (Sanders, 2020), indicating a dire need for adolescent adversity intervention.

\section{Latinx Immigrant ACEs as a Health Paradox}

The unique finding of lower frequencies of ACEs among Latinx immigrant children in the USA is a paradoxical finding considering the many challenges that most immigrant families face in the process of adapting to their new life in the USA. The most pervasive and unique stressor to immigrants is the anxiety of deportation. Immigrant parents and children show heightened stress levels due to fear of separation from family, regardless of their immigration status (Vaughn et al., 2015). This means that the anxieties of deportation exist even among immigrant families who reside legally within the USA (Vaughn et al., 2015). Further stresses result from actual detainment or deportation of immigrant parents. Immigrant children who do experience parental deportation experience distress from a traumatic perceived dissolution of their family (Vaughn et al., 2015). Children of detained or deported parents are shown to have higher risks of mental disorders like depression, anxiety, and attention disorders, as well as higher rates of negative self-esteem, delays in speech, compromised academic performances, and relational difficulties (Flores \& Salazar, 2017). Another deportation-related stressor is when children who are legal US citizens are unable to remain in the country due to parental deportation (Vaughn et al., 2015).

The extent of stressors unique to immigrant families can be examined further to analyze this paradox. Immigrant parents experience cultural and language barriers as well as higher rates of low socioeconomic statuses (Vaughn et al., 2015). Further stressors among immigrants include acculturation, loss of heritage, familial stability concerns, and financial instability (Vaughn et al., 2015). The threat of deportation contributes to an underuse and general lack of knowledge about social services. For example, undocumented parents are less likely to receive food and childcare subsidies and health insurance (Passel \& D’Vera, 2011). Fears of deportation and discrimination can deter both immigrant mothers and children from reporting abuse to officials or seeking help (Vaughn et al., 2015). Despite these higher rates of poverty and familial stress due to US Immigration and Customs Enforcement (ICE) threats, discrimination, and cultural barriers, data shows lower ACEs reports among Latinx immigrant children than nonimmigrant children (Caballero et al., 2017). 


\section{Failure of ACEs to Capture Latinx Immigrant Childhood Adversity}

The health paradox may be a result of traditional ACEs measures failing to capture immigrant-specific experiences of adversity (Cronholm et al., 2015). One study found that $13.9 \%$ of participants who experienced adversity were only identified through an expanded definition and category of ACEs, and these participants would have gone unrecognized if only the conventional measures of ACEs had been used (Cronholm et al., 2015). Thus, the contradictory findings of increased adversity and a low frequency of ACEs among immigrant children may not reflect a true health advantage in this population, but rather the failure of traditional ACE measures to represent adverse experiences specific to immigrants, for example, the ten experiences of adversity that ACE measures does not include adversity unique to immigrant children, like ICE arrests or deportations of parents or guardians, being a victim of or witnessing ICE arrests or raids, parent or guardian separation because of migration, and experiencing anti-immigrant discrimination (Crouch et al., 2019; Flores \& Salazar, 2017). Even the ten ACEs may simply be underreported within immigrants. Fears of deportation and discrimination can deter both immigrant mothers and children from reporting abuse, which can contribute to both inaccurate reports of ACEs and a lack of understanding as to what ACEs look like in immigrant children (Vaughn et al., 2015).

Other measures exist to capture experiences of adversity, trauma, and toxic stress beyond the traditional ACEs. Table 2 depicts these experiences of adversity unique to immigrants that are not captured by the traditional 10 ACEs (Table 2).

Children who fear or experience separation from their parents are shown to exhibit toxic stress (Vargas et al., 2017). Research shows that immigrant children who have witnessed immigration raids can experience toxic stress that impacts their

Table 2 Immigrant experiences of adversity outside of the ACEs framework

\begin{tabular}{|c|c|}
\hline Experience & Effects \\
\hline Discrimination (xenophobia, racism) & Anxiety, depression, PTSD \\
\hline Fear of deportation & Anxiety, mental health syndromes \\
\hline $\begin{array}{l}\text { Fear of deportation of parents, } \\
\text { relatives, and friends (separation } \\
\text { anxiety) }\end{array}$ & Avoidance of social and economic activities, toxic stress \\
\hline Lack of documentation & $\begin{array}{l}\text { Anxiety, denial of emergency care, exclusion from schooling, fear } \\
\text { of participating in public programs, lack of daily necessities, } \\
\text { threats of violence }\end{array}$ \\
\hline $\begin{array}{l}\text { Living in states with anti-immigra- } \\
\text { tion policies (fear of association) }\end{array}$ & $\begin{array}{l}\text { Anxiety, job and financial insecurity, poor health outcomes, poor } \\
\text { housing conditions }\end{array}$ \\
\hline Separation from parents & $\begin{array}{l}\text { Attachment disorders, behavioral issues, chronic illnesses, } \\
\text { developmental delays, lack of supportive adult relationships, } \\
\text { substance abuse, toxic stress }\end{array}$ \\
\hline Witness of immigration raids & Asthma, developmental delays, toxic stress, obesity \\
\hline
\end{tabular}

Sources: Capps \& Fix, 2020; Crouch et al., 2019; Flores \& Salazar, 2017; Santhanam, 2018; Vargas et al., 2017 
development and health outcomes, including delays in development, asthma, obesity, and other concerns related to health (Vargas et al., 2017). Research also shows that the longer children are separated from their deported or detained mothers, the more extreme toxic stress and its risks become (Santhanam, 2018). As the primary way to lessen the impacts of toxic stress is through adult relationships, separation from one's parents is both the cause and lost relief to this extreme adversity (Santhanam, 2018).

Latinx teenagers exhibit higher incidences of anxiety and other mental health symptoms because of discrimination and deportation fears (Capps \& Fix, 2020). One recent study of a sample of Latinx students found that over half exhibited clinically diagnosable levels of post-traumatic stress disorder (PTSD), anxiety, and depression; over three-quarters reported high levels of anxiety; and on average, students reported having experienced over seven "traumatic life events" (witnessing or experiencing assault, losing a loved one, separation from parent, etc.) (Capps \& Fix, 2020). Clearly, the toxic stress resulting from deportation anxiety is prevalent within Latinx immigrant families, yet first generation Latinx immigrant children show lower ACE scores on average, suggesting that ACE questionnaires are unfit to capture the adversity specific to immigrant families.

\section{Protective factors to ACEs in Latinx Communities}

After reviewing the theory that ACEs are not accurately reported for immigrant children to explain low ACE scores (Cronholm et al., 2015), a second theory can also be explored. Another interpretation of the health paradox is that low ACEs reports suggest unmeasured factors that buffer children in immigrant families from ACEs exposure (Van Hook et al., 2013). There may be potential protective factors specific to immigrant families that nonimmigrant children do not experience. One source of evidence for this theory is that a low prevalence of ACEs is not the only health paradox among Latinx immigrants. For example, children of immigrants have lower rates of infant mortality and low birth weight than expected based on their average socioeconomic status (Van Hook et al., 2013). Children of immigrants also have lower rates of developmental delays, learning disabilities, and poor health outcomes like allergies, asthma, obesity, smoking, and substance abuse compared to children of native families (Van Hook et al., 2013).

Latinx immigrant children's lower prevalence of ACEs despite higher rates of poverty is theorized to be associated with the self-selection qualities of first-generation immigrants (Crouch et al., 2019). Immigrant parents must be skilled in facing challenges like learning a novel language, navigating financial and legal differences, acculturation, building community, forming relationships, and more (Van Hook et al., 2013). Biculturalism is an adaptive response that consists of retaining positive values from one's origin culture and adapting positive values from the new culture, creating a "best of both worlds" scenario for immigrant parental values (American Academy of Pediatrics, 2020). Immigrant parents gaining experience in solving challenges as well as retaining the protective aspects of their original culture can instill resilience in their children (Van Hook et al., 2013). Resilient parents are 
shown to promote resiliency in their own children (American Academy of Pediatrics, 2020), which is a key determinant of positive health outcomes despite childhood adversity.

The families themselves often have qualities that serve as protective factors. Immigrant families demonstrate increased social and material support because of strong familial networks (Van Hook et al., 2013). They often exhibit higher cohesion, communication, and support, and they more often consist of two parents (American Academy of Pediatrics, 2020). Parents who themselves are immigrants are more likely to instill values of work ethic and high aspirations among their children (American Academy of Pediatrics, 2020). Further, the values of connection and community within Latinx culture are extremely beneficial towards both gaining support and promoting resilience (Van Hook et al., 2013). Immigrants who participate in an engaging community of immigrants from their origin country have better mental health outcomes than those who do not (American Academy of Pediatrics, 2020).

These protective factors (self-selection qualities, biculturalism, resilience, strong familial networks, two parent households, values of connection and community, etc.) are shown to fade with acculturation, explaining the increased prevalence of ACEs among later generations of immigrants (Van Hook et al., 2013). For example, high health outcomes among Latinx immigrants is related to retainment of origin country health practices, like daily cultural health-promoting routines and low rates of maternal substance abuse (Van Hook et al., 2013). As assimilation occurs, immigrants lose some of these health-promoting practices, providing explanation for earlier generation immigrants having better developmental and health outcomes (Van Hook et al., 2013). As immigrants lose connection to their familial, ethnic, and immigrant support networks, these strong social connections and their benefits lessen in strength (Van Hook et al., 2013).

\section{Consequences of ACEs in Child Development and Lifelong Health}

The importance of the findings of this review (the potential failure of ACEs to accurately capture Latinx experiences of adversity as well as the need to classify the COVID-19 pandemic as an ACE) can be emphasized through the connection of childhood experiences of adversity and toxic stress to lifelong health outcomes. Particularly, as the COVID-19 pandemic is a public health epidemic that disproportionately affects Latinx communities, this connection between adversity and health outcomes has drastic implications for the well-being of children in the USA. Scientific research by the National Scientific Council on the Developing Child (2020) shows that children's early experiences with adversity and toxic stress have both immediate and long-lasting implications for their physiological, psychological, and social health without adequate protective factors.

Adversity is shown to impair the development of various biological systems as a result of abnormally elevated levels of stress hormones for long periods of time (National Scientific Council of the Developing Child, 2005/2014). Nilson and colleagues (2019) found that 18-month-olds exposed to adversity were more likely to have delays in cognitive development, behavioral regulation, and attention, and 
they were more likely to display symptoms of anxiety or depression. These delays can be expressed through poor development of immune and neuroendocrine systems (Mersky et al., 2013). Four or more ACEs experienced in infancy is associated with a higher risk of developmental delays and a chronic health condition due to an underdeveloped immune system in the first five years of life (McKelvey et al., 2017).

By age 11, there are significant associations between ACEs experienced pre-toddlerhood and obesity, respiratory issues, and a decreased parental perception of the child's health (McKelvey et al., 2017). Consequences of adversity can be connected to causes of death as those who experienced four or more ACEs were 22 times as likely to die as a result of injury, abuse, or suicide compared to children with zero ACEs (Grey et al., 2019). By the age of 18, the effects of early childhood adversity can result in higher tendencies toward emotional dysregulation and impulsivity, which may be exhibited through maladaptive behavior patterns relating to substance abuse and risky sexual behavior in adulthood (Espeleta et al., 2018). Later in life, greater levels of adversity in childhood are associated with lower ratings of adults' reported health and life satisfaction, high-risk health behaviors, chronic diseases and mental disorders, and premature death (Mersky et al, 2013, Flores et al., 2017, Espeleta et al., 2018).

The effects of ACEs are not confined to the individual who experienced adversity. The health and behavior patterns of parents have immediate impacts on their children, and ACEs impact familial qualities like educational attainment, employment status, and overall income and wealth (Mersky et al., 2013). Furthermore, there is evidence of intergenerational effects of ACEs and trauma through links between parental ACE scores and child exposure to ACEs, coined as "secondary trauma" by Mersky et al. (2013). These effects of ACEs can be displayed through intergenerational transitions of attachment types, as parent-child interactions and parental mental health are a large determinant of a child's attachment style (Cittern et al., 2018). If a child is to transition from a secure to insecure attachment style, it is often linked to higher ACE scores or traumatic experiences of either the parent or the child (Cittern et al., 2018).

The National Scientific Council on the Developing Child (2014) provides insight into the impacts of adversity on development in its exploration of toxic stress. Toxic stress is the result of extended activation of the physiological stress response system and the lack of protective factors like supportive adult relationships (National Scientific Council of the Developing Child, 2005/2014). Moreover, toxic stress is described as strong, frequent, and/or prolonged adversity, which includes those experiences coined by Felitti and colleagues as ACEs (Felitti et al., 1998). The intersection between ACEs and toxic stress includes abuse and violence, extended neglect, caregiver substance abuse and/or mental illness, and an accumulation of family economic burdens (National Scientific Council on the Developing Child, 2014). Thus, toxic stress can be thought of as a child's biological response to ACEs that directly affects development.

In examining ACEs and toxic stress, it is key to note that adversity in childhood is not necessarily a dooming sentence of life-long ill health. Psychological resilience refers to the ability to cope and adapt to adversity, trauma, or crises by decreasing stress through mental or emotional strategies (National Scientific Council on the 
Developing Child, 2005/2014). Adversity can encourage an individual to form resilient tendencies, build social support networks, and develop goal efficacy (Thomson \& Jaque, 2017), but only with the proper environments, relationships, and support to promote resiliency. These findings demonstrate the importance of building resiliency to negate the negative impacts of ACEs and have their strengths in identifying clear intervention pathways to encourage protective factors.

\section{Evidence-Based Interventions to Prevent ACEs}

The interaction of childhood adversity and lifelong health is essential to keep in mind when analyzing existing and recommended interventions to reduce the negative impact of ACEs on children through available literature. Promoting resiliency in Latinx immigrant children would benefit lifelong immune health and overall well-being (Shonkoff and Williams, 2020), thus offering protective factors against COVID-19 as well as other health disparities related to ACEs. The evidence-based interventions that are discussed in this section include the promotion of resiliency through healthy adult relationships, policies that screen for ACEs early on in a child's life, trauma-informed care and innovative treatment programs, and strengthening existing protective services through financial and political support.

Resiliency can be encouraged through multiple factors like high educational attainment, secure attachment styles, and involving oneself in meaningful activities (Thomson \& Jaque, 2017). The single most influential characteristic of a child's life to promote resiliency to adversity is external intervention through a supportive, responsive adult relationship (National Scientific Council on the Developing Child, 2005/2014). Research has found that among participants with four or more ACEs, over half were classified as resilient (Thomson \& Jaque, 2017), and further interventions that focus on promoting resiliency have the potential to widen this margin.

Policies and programs that identify, prevent, and ameliorate the impacts of ACEs are needed to benefit development and health outcomes of all children. One of the most effective categories of interventions are those that work to screen for ACEs as early in the child's life as possible. A widely argued policy change to benefit early identification of adversity is the universal screening of ACEs in pediatric primary care (Bryant et al., 2020). Various states have adopted policies to aid this screening process with the goals of reducing the incidence of ACEs in future generations. Since 2009, 48 states have included at least one question concerning ACEs on their Behavioral Risk Factor Surveillance System (BRFSS) survey (Cooper \& Hanlon, 2020). Universal pediatric screening for ACEs has become standard in California (Bryant et al., 2020) with goals of reducing the number of ACEs by half (Cooper \& Hanlon, 2020). North Carolina has added a health indicator to measure ACEs as part of a comprehensive plan to reduce the number of ACEs by improving the social determinants of health through economic opportunities, trauma-informed support, domestic abuse interventions, and increased access to parenting programs (Cooper \& Hanlon, 2020).

The adoption of innovative treatment and screening processes has increased the capacity of healthcare professionals to work with adversity. While the pandemic has 
worsened experiences of adversity, it has also inspired innovative health policies and practices, like the use of "contact tracers" to identify ACEs promptly and mitigate the long-term impacts of COVID-19 related adversity (Sanders, 2020). Pediatric offices have developed innovative methods to treat toxic stress like trauma-informed cognitive-behavioral therapy and eye movement desensitization and reprocessing therapy (Bryant et al., 2020). All pediatric medical offices should be trained in trauma-informed care, integrate mental-health services, and operate with a socioecological framework of health (Sanders, 2020). Primary care should attend to social needs like healthcare access, food and housing security, domestic violence, and unemployment through partnerships with local agencies that attend to these needs (Sanders, 2020).

There is a need for investment in systems beyond health care that address childhood adversity. Increasing financial support to effective child protective services and welfare systems will aid their expertise in identifying and intervening situations of abuse and neglect (Bryant et al., 2020). Increased attention to Latinx youth specific mental health concerns in schools and youth institutions through culturally appropriate counseling and intervention services will address the structural inequities of adversity (Capps \& Fix, 2020). Shifts in immigration and deportation policy like restoring and improving the Deferred Action for Childhood Arrivals (DACA) program will contribute to lessening Latinx immigrant children's anxiety (Capps \& Fix, 2020). Children with diverse backgrounds like those in immigrant families respond better to screening using more inclusive measures of adversity and referral to mental and pediatric health professionals, legal services, and resources to address social determinants of health (Sanders, 2020).

\section{Conclusions and Recommendations}

In applying the ACEs framework to Latinx populations in the USA, relevant findings concerning a potential failure of ACEs to accurately capture Latinx experiences of adversity were highlighted, as well as the need to classify the COVID-19 pandemic as an ACE. The COVID-19 pandemic has shown to exacerbate childhood adversity and toxic stress and has disproportionately harmed Latinx communities. The COVID-19 pandemic, as an additional ACE, has impacted the health and well-being of Latinx populations in the USA, particularly by exacerbating preexisting health and other related inequities in this population. Funding agencies should prioritize supporting research on the topic of COVID-19 pandemic as a potential ACE and its effect on Latinx children's development to generate knowledge in order to orient interventions based on empirical data.

The traditional measures of ACEs can miss experiences of adversity unique to diverse populations, including immigrants. The theory that the paradox of low ACEs among immigrant populations is a result of the failure of ACEs to capture Latinx immigrant childhood adversity was supported after analyzing findings of the reviewed literature. This phenomenon is an important finding in that it may represent the failure of ACEs to accurately measure adversity and protect minority children. Research may also miss diverse cultural factors that offer unique protective 
factors for ACEs in this population. Latinx and immigrant children must be prioritized and kept at the forefront of future research and interventions by accounting for their diversity, as well as their cultural, linguistic, and ecological characteristics and realities. There is extensive and unmeasured value of expanding adversity research to include underrepresented contexts.

Future ACEs related research regarding this gap must have two directions. First, the potential protective factors of the cultures of immigrant families should be explored to aid development of the most effective policies and techniques for adversity intervention considering the paradox observed in ACEs frequency among Latinx children in the USA (Caballero et al., 2017). Second, the conventional ACEs should be evaluated in their effectiveness of including all populations and potentially expanded to be inclusive of all sociodemographic groups, particularly immigrants and their unique experiences. ACEs must be inclusive of the toxic stress that arises from diverse experiences, like parental arrests/deportation or witnessing/ experiencing raids (Flores \& Salazar, 2017). Interventions like those of North Carolina that focus on reducing ACEs by improving the social determinants of health through economic opportunities, trauma-informed support, domestic abuse interventions, and increased access to parenting programs (Cooper \& Hanlon, 2020) expand beyond the ACEs framework and thus are a replicable example of a comprehensive program inclusive of immigrant-related factors that promote toxic stress in children.

ACEs can be prevented with the implementation of evidence-based, holistic interventions. Avoidable experiences of abuse, household challenges, and neglect are shown to have significant negative impacts on development with both short and long-term impacts on physical, mental, and social health. Through proper interventions, policy, and funding, children can be protected to benefit their immediate well-being and their futures. The findings of evidence-based interventions to ACEs should be applied to the everyday world through guiding effective interventions to protect children from experiencing adversity and developing treatments that negate the long-term negative effects of ACEs through responsive, supportive adult relationships. Working to reconceptualize early childhood policies and programs to strengthen lifelong health offers a proactive approach to building lifelong resiliency (National Scientific Council on the Developing Child, 2020). Addressing structural inequalities by strengthening early childhood foundations of lifelong health will address inequalities in disease susceptibility, adverse childhood experiences, and health outcomes (Shonkoff and Williams, 2020). It may not always be possible to intervene with abuse before it happens, especially during challenging times like we are currently experiencing during the COVID-19 pandemic. However, we can create environments that promote resilience, resources, and strong foundations of health to negate the negative impacts of adversity in childhood.

\section{Limitations}

The limitations of this narrative literature review include that studies concerning Latinx youth often did not consider immigrant status, nor emphasize potential protective factors within immigrant families, or critically analyze the 
effectiveness of the conventional ACEs parameters (Felitti et al., 1998). These limitations often concern a lack of emphasis on potential confounders or relevant determinants, such as immigration status and a generation level. Future publications should take care to avoid these biases and bridge the gaps of unexplored knowledge. As a narrative literature review, the format experiences limitations in that it may not meet as rigorous of criteria to mitigate bias and offer conclusions. As an explorative process, the procedure holds less explicit criteria for article selection and evaluation of validity. Further, as the impacts of COVID-19 pandemic were considered as an ACE in this study, the nature of data concerning this current event can be lacking in peer-reviewed, empirical studies, and instead rely on current events, government publications, and comparisons to similar historical events.

Author Contribution Not applicable.

Availability of Data and Materials All utilized data is available through public databases.

Code Availability Not applicable.

Declarations

Ethics Approval Not applicable.

Consent to Participate Not applicable.

Consent for Publication This work has not been published before; it is not under consideration for publication anywhere else; its publication has been approved by all co-authors. The publisher will not be held legally responsible should there be any claims for compensation.

Conflict of Interest The authors declare no competing interests.

\section{References}

American Academy of Pediatrics. (2020). Immigrant child health toolkit. Retrieved from https:// www.aap.org/en-us/advocacy-and-policy/aap-health-initiatives/Immigrant-Child-Health-Toolkit/ Pages/Immigrant-Child-Health-Toolkit.aspx

APA (2020). How COVID-19 may increase domestic violence and child abuse. Retrieved from: https://www.apa.org/topics/covid-19/domestic-violence-child-abuse.

Branson-Potts, H., Reyes-Velarde, A., Stiles, M., \& Campa, A. J. (2020). The price of being 'essential': Latino service workers bear brunt of coronavirus. Retrieved from https://www.latimes. com/california/story/2020-05-17/latino-essential-workers-coronavirus

Bryant, D. J., Oo, M., \& Damian, A. J. (2020). The rise of adverse childhood experiences during the COVID-19 pandemic. Psychological Trauma: Theory, Research, Practice, and Policy, 12(S1), S193-S194. https://doi.org/10.1037/tra0000711

Caballero, T. M., Johnson, S. B., Buchanan, C., \& DeCamp, L. R. (2017). Adverse childhood experiences among Hispanic children in immigrant families versus us-native families. Pediatrics, 140(5), e20170297. https://doi.org/10.1542/peds.2017-0297

Capps, R., \& Fix, M. (2020). How the fear of immigration enforcement affects the mental health of Latino youth. Migration Policy Institute. 
Center on the Developing Child (2007). The science of early childhood development (InBrief). Retrieved from www.developingchild.harvard.edu

Chowdhury, S. M. (2020). Conflict in the Time of (Post-) Corona: Some assessments from behavioral economics. Peace Economics, Peace Science, \& Public Policy, 26(3), 1-10. https://doi-org.libproxy. clemson.edu/https://doi.org/10.1515/peps-2020-0052

Cittern, D., Nolte, T., Friston, K., \& Edalat, A. (2018). Intrinsic and extrinsic motivators of attachment under active inference. PLOS ONE, 13(4). https://doi-org.libproxy.clemson.edu/https://doi.org/10. 1371/journal.pone.0193955

Cooper, R. \& Hanlon, C. (2020). States' efforts to address adverse childhood experiences are critical during COVID-19. National Academy for State Health Policy. Retrieved from https://www.nashp.org/ states-efforts-to-address-adverse-childhood-experiences-is-critical-during-covid-19/

Cronholm, P. F., Forke, C. M., Wade, R., Bair-Merritt, M. H., Davis, M., Harkins-Schwarz, M., et al. (2015). Adverse Childhood Experiences: Expanding the concept of adversity. American Journal of Preventive Medicine, 49(3), 354-361. https://doi.org/10.1016/j.amepre.2015.02.001.

Crouch, E., Probst, J. C., Radcliff, E., Bennett, K. J., \& McKinney, S. H. (2019). Prevalence of adverse childhood experiences (ACEs) among US children. Child Abuse \& Neglect, 92, 209-218. https:// doi.org/10.1016/j.chiabu.2019.04.010

Ellis, W. R., \& Dietz, W. H. (2017). A new framework for addressing adverse childhood and community experiences: The building community resilience model. Academic Pediatrics, 17(7S), S86-S93. https://doi.org/10.1016/j.acap.2016.12.011

Espeleta, H. C., Brett, E. I., Ridings, L. E., Leavens, E. L. S., \& Mullins, L. L. (2018). Childhood adversity and adult health-risk behaviors: Examining the roles of emotion dysregulation and urgency. Child Abuse \& Neglect, 82, 92-101. https://doi-org.libproxy.clemson.edu/https://doi.org/ 10.1016/j.chiabu.2018.05.027

Felitti, V. J., Anda, R. F., Nordenberg, D., Williamson, D. F., Spitz, A. M., Edwards, V., Koss, M. P., \& Marks, J. S. (1998). Relationship of childhood abuse and household dysfunction to many of the leading causes of death in adults: The adverse childhood experiences (ACE) study. American Journal of Preventive Medicine, 14, 245-258. https://doi.org/10.1016/S0749-3797(98)00017-8

Flores, G., \& Salazar, J. C. (2017). Immigrant Latino children and the limits of questionnaires in capturing adverse childhood events. Pediatrics, 140(5), e20172842. https://doi.org/10.1542/peds. 2017-2842

Gelatt, J., Batalova, J., \& Capps, R. (2020). An early readout on the economic effects of the COVID-19 crisis: Immigrant women have the highest unemployment. Migration Policy Institute.

Grey, H. R., Ford, K., Bellis, M. A., Lowey, H., \& Wood, S. (2019). Associations between childhood deaths and adverse childhood experiences: An audit of data from a child death overview panel. Child Abuse \& Neglect, 90, 22-31. https://doi-org.libproxy.clemson.edu/https://doi.org/10. 1016/j.chiabu.2019.01.020

Lawson, M., Piel, M. H., \& Simon, M. (2020). Child maltreatment during the COVID-19 pandemic: Consequences of parental job loss on psychological and physical abuse towards children. Child Abuse \& Neglect, 110(Pt 2), 104709. https://doi.org/10.1016/j.chiabu.2020.104709

Loria, H., \& Caughy, M. (2018). Prevalence of adverse childhood experiences in low-income Latino immigrant and nonimmigrant children. The Journal of Pediatrics, 192, 209-215. https://doi-org.libproxy.clemson.edu/https://doi.org/10.1016/j.jpeds.2017.09.056

Maguire-Jack, K., Lanier, P., \& Lombardi, B. (2020). Investigating racial differences in clusters of adverse childhood experiences. The American Journal of Orthopsychiatry, 90(1), 106-114. https:// doi.org/10.1037/ort0000405

McKelvey, L. M., Saccente, J. E., \& Swindle, T. M. (2019). Adverse childhood experiences in infancy and toddlerhood predict obesity and health outcomes in middle childhood. Childhood Obesity, 15(3), 206-215. https://doi-org.libproxy.clemson.edu/https://doi.org/10.1089/chi.2018.0225.

Mersky, J. P., Topitzes, J., \& Reynolds, A. J. (2013). Impacts of adverse childhood experiences on health, mental health, and substance use in early adulthood: A cohort study of an urban, minority sample in the US. Child Abuse \& Neglect, 37(11), 917-925. https://doi-org.libproxy.clemson.edu/https://doi. org/10.1016/j.chiabu.2013.07.011.

National Scientific Council on the Developing Child (2005/2014). Excessive stress disrupts the architecture of the developing brain: Working paper no. 3. Updated Edition. Retrieved from www.devel opingchild.harvard.edu. 
National Scientific Council on the Developing Child (2020). Connecting the brain to the rest of the body: Early childhood development and lifelong health are deeply intertwined. Working paper No. 15. Retrieved from www.developingchild.harvard.edu

Nilsson, K. K., Landorph, S., Houmann, T., Olsen, E. M., \& Skovgaard, A. M. (2019). Developmental and mental health characteristics of children exposed to psychosocial adversity and stressors at the age of 18-months: Findings from a population-based cohort study. Infant Behavior \& Development, 57. https://doi-org.libproxy.clemson.edu/https://doi.org/10.1016/j.infbeh.2019.04.001

Noe-Bustamante, L., Mora, L., \& Lopez, M. (2020). Latinx used by just 3\% of U.S. Hispanics. About one-in-four have heard of it. Retrieved October 24, 2020, from https://www.pewresearch.org/hispa nic/2020/08/11/about-one-in-four-u-s-hispanics-have-heard-of-latinx-but-just-3-use-it/.

Panchal, N., Kamal, R., Orgera, K., Cox, C., Garfield, R., Hamel, L.,...Chidambaram, P. (2020). The implications of COVID-19 for mental health and substance use. Retrieved from https://www.kff.org/ health-reform/issue-brief/the-implications-of-covid-19-for-mental-health-andsubstance-use/.

Parolin, Z. (2021). What the COVID-19 pandemic reveals about racial differences in child welfare and child well-being: An introduction to the special issue. Race and Social Problems, 1-5. https://doiorg.libproxy.clemson.edu/https://doi.org/10.1007/s12552-021-09319-2.

Raviv, T., Warren, C. M., Washburn, J. J., Kanaley, M. K., Eihentale, L., Goldenthal, H. J., Russo, J., Martin, C. P., Lombard, L. S., Tully, J., Fox, K., \& Gupta, R. (2021). Caregiver perceptions of children's psychological well-being during the COVID-19 pandemic. JAMA Network Open, 4(4), e2111103. https://doi-org.libproxy.clemson.edu/https://doi.org/10.1001/jamanetworkopen.2021. 11103.

Sanders, L. M. (2020). Is COVID-19 an adverse childhood experience (ACE): Implications for screening for primary care. The Journal of Pediatrics, 222, 4-6. https://doi.org/10.1016/j.jpeds.2020.05.064

Santhanam, L. (2018). How the toxic stress of family separation can harm a child. Retrieved from https:// www.pbs.org/newshour/health/how-the-toxic-stress-of-family-separation-can-harm-a-child.

Shonkoff, J. P., \& Williams, D. R. (2020, April 27). Thinking about racial disparities in COVID-19 impacts through a science-informed, early childhood lens. Retrieved from https://developingchild. harvard.edu/thinking-about-racial-disparities-in-covid-19-impacts-through-a-science-informedearly-childhood-lens/.

Taub, A. (2020). A new COVID-19 crisis: Domestic abuse rises worldwide. New York Times. Retrieved from https://www.nytimes.com/2020/04/06/world/coronavirus-domestic-violence.html.

Tener, D., Marmor, A., Katz, C., Newman, A., Silovsky, J. F., Shields, J., \& Taylor, E. (2020). How does COVID-19 impact intrafamilial child sexual abuse? Comparison analysis of reports by practitioners in Israel and the US. Child abuse \& neglect, 104779. Advance online publication. https://doi.org/10. 1016/j.chiabu.2020.104779

Thomson, P., \& Jaque, S. V. (2017). Adverse childhood experiences (ACE) and adult attachment interview (AAI) in a non-clinical population. Child Abuse \& Neglect, 70, 255-263. https://doi-org.libproxy.clemson.edu/https://doi.org/10.1016/j.chiabu.2017.06.001.

Van Hook, J., Landale, N. S., \& Hillemeir, M. M. (2013). Is the United States bad for children's health? Risk and resilience among young children of immigrants. Migration Policy Institute.

Vargas, E. D., Sanchez, G. R., \& Juárez, M. (2017). Fear by association: Perceptions of anti-immigrant policy and health outcomes. Journal of Health Politics, Policy and Law, 42(3), 459-483. https://doi. org/10.1215/03616878-3802940

Vaughn, M., Salas-Wright, C., Huang, J., Qian, Z., Terzis, L., \& Helton, J. (2015). Adverse childhood experiences among immigrants to the United States. Journal of Interpersonal Violence. 32.https:// doi.org/10.1177/0886260515589568.

Vespa, J., Medina, L., \& Armstrong, D.M. (2020). Demographic turning points for the United States: Population projections for 2020 to 2060. United States Census Bureau. Retrieved from https://www. latinopolicyforum.org/publications/briefs/document/Poder_Ed-FINAL.pdf.

Passel, J.S. \& D'Vera C. (2011). Unauthorized immigrant population: National and state trends, 2010. Pew Hispanic Center. Retrieved from www.pewhispanic.org.

Zaami, S, Marinelli, E, \& Varì, M.R. (2020). New trends of substance abuse during COVID-19 pandemic: An international perspective. Front. Psychiatry, https://doi.org/10.3389/fpsyt.2020.00700.

Publisher's Note Springer Nature remains neutral with regard to jurisdictional claims in published maps and institutional affiliations. 


\section{Authors and Affiliations}

\section{Natalie Claypool ${ }^{1}$. Arelis Moore de Peralta ${ }^{2}$}

Natalie Claypool

nclaypo@g.clemson.edu

1 Clemson University, Clemson, SC 29634, USA

2 Department of Languages, Clemson University, 504 Strode Tower, Clemson, SC 29634, USA 